\title{
DESIGN AND PERFORMANCE EVALUATION OF NEXT GENERATION CLUTCH CONTROL VALVE
}

\author{
Michael Erhard*, Jörg Schneider, Jan Julius Stein \\ Thomas Magnete GmbH, Innomotion Park 3, 57562 Herdorf \\ * Corresponding author: Tel.: +49 2744 929103; E-mail address: michael.erhard@thomas-magnete.com
}

\begin{abstract}
Pressure reducing valves perform various control tasks in mobile machines. In many cases contamination sensitive environments require pilot-operated valves, because of its more robust performance. On the other hand, dynamic valve behavior can be critically in certain applications. Overcoming the restrictions resulting from stand-alone test rig attempts, suitable simulation principles are nowadays the state of art in valve design. Unsuitable model approaches and missing consideration of the application may result in wrong design decisions. This paper discusses two important modeling issues, in particular the solenoid and spool leakage behavior, in combination with the connected system under control, a pressure-actuated clutch. It is shown, that even for standard proportional valves a critical view on customers' excitation strategy and site of installation decides on success or failure of an already used design variant.
\end{abstract}

Keywords: Pressure Valve, Holistic Simulation, Clutch Control

\section{INTRODUCTION}

Proportional pressure reducing and relieving valves for pilot applications are together with pilot-operated variants for clutch or fan drive control the main business segments at Thomas Magnete $\mathrm{GmbH}$ in the field of mobile machinery. Current developments are aimed among other things at mechatronic subsystems with integrated electronic and possible electro-mechanical actuation. Nevertheless, the continuous development of existing products is a guarantee for further success in the market.

Typical valve variants in use consist of a solenoid mechanical connected to a valve spool, whereas the regulated pressure is directly balanced by the solenoid or a so called pressure pin is inserted as an intermediate element. In contrast to these so-called direct-operated valves, pilot-operated ones uses a hydraulic coupling between the valve spool and the solenoidcontrolled pilot stage. This increase in physical complexity may cause problems in some applications, especially under dynamic aspects. Besides step responses, continuous transient input variations as they occur in clutch control applications are also significant. Under unfavorable boundary conditions and fast transients, inadequate pressure control can sometimes be observed. Figure 1 illustrates the above mentioned product family with the focused valve at the bottom.

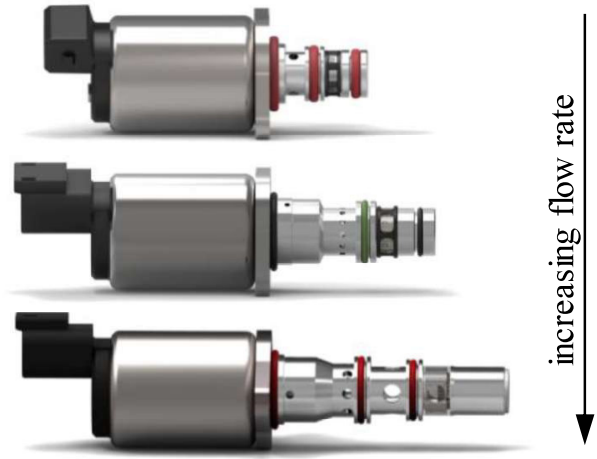

Figure 1: Typical proportional pressure reducing valves with different flow capacity

The article is organized as follows: The $2^{\text {nd }}$ section discusses necessary modeling challenges with special focus on solenoid and spool leakage behavior for a reference valve design. A complete simulation model for typical valve characteristic curves and controller performance, including the pressure-actuated clutch, is mentioned. In the $3^{\text {rd }}$ section modeling basics are used to validate test rig observations from the reference valve design with special focus on pre-developed modeling 
approaches. Resulting from this, requirements for a further development avoiding poor dynamic pressure control behavior are identified. The $4^{\text {th }}$ section explains simulation-based design changes and their validation on the test rig. The $5^{\text {th }}$ section summarizes the content of this article.

\section{MODELING CHALLENGES}

From a control engineering point of view, proportional pressure reducing valves are closedloop controllers for an output pressure with an electric input specification under a hydraulic disturbance. Otherwise, these electro-hydraulic valves yield from a mathematical perspective an initial value problem arising from a system of stiff nonlinear differential equations as indicated in Equation 1.

$\dot{\vec{x}}=\mathrm{f}(\vec{x}, t)$

Thus the modeling task is clearly defined. Relevant equations for the different physical domains have to be combined according to the functional structure shown in Figure 2 resulting in a dynamic model for the entire valve.

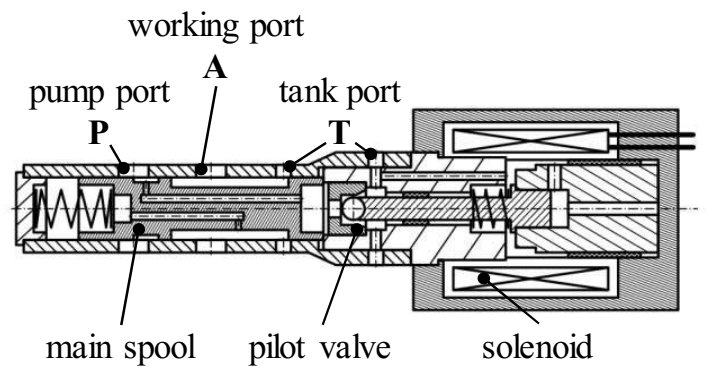

Figure 2: Sketch of investigated valve and its crosselementary interactions

This model must fulfill the requirements from the controller site meaning besides boundary conditions only time-dependent input and disturbance variations are allowed as "external" model inputs. The controlled pressure results only from the intrinsic closed-loop controller properties.

\subsection{Solenoid dynamics}

A main component of the entire valve is the electro-magneto-mechanical transducer. A holistic approach should include its position- and current-dependent force characteristic as well as its transient relations between voltage, current and force respectively. Thus the solenoid alone represents a MIMO system with two inputs and two outputs [1]. Various authors have investigated different approaches to model these system structure based on flux linkage [1], coupled reluctance networks [2] or electric equivalent circuits [3,4]. Essential differences arise from dealing with eddy currents and moving induction. The parameter for these models are often determined with latest finite-elementmethods.

Starting point of the dynamic model development is the electric equivalent circuit approach including eddy currents and moving induction. The necessity results from experimental observations with the reference design shown in the $3^{\text {rd }}$ section whereas different excitation strategies in solenoid current control mode strongly influence accuracy and smoothness of the transient closed-loop pressure control.

Benchmarking several model structures and parameter schemes yield a promising result illustrated in Figure 3 with governing Equations 2 - 4. This approach models the nonlinear time lag between voltage $U_{\text {coil }}$ and current $i_{\text {coil }}$ taking into account the additional time lag between current and force $F_{\text {arm }}$ because of eddy effects $\left(i_{e d d y_{-} f}+i_{e d d y_{-} s}\right)$.

$$
\begin{aligned}
\frac{\partial i_{\text {coil }}}{\partial t}= & \frac{1}{\frac{\partial \Psi}{\partial i_{\text {coil }}}} \cdot\left(U_{\text {coil }}-R_{\text {coil }}\right. \\
& \cdot\left(i_{\text {coil }}+i_{\text {eddy_f }}+i_{\text {eddy_s }}\right) \\
& \left.-\frac{\partial \Psi}{\partial x_{\text {arm }}} \cdot \frac{\partial x_{\text {arm }}}{\partial t}\right)
\end{aligned}
$$

$$
\begin{aligned}
\frac{\partial i_{e d d y_{-} f}}{\partial t}= & \frac{1}{L_{e d d y_{-} f}} \cdot\left(U_{\text {coil }}-R_{\text {coil }}\right. \\
& \cdot\left(i_{\text {coil }}+i_{e d d y_{-} f}+i_{e d d y_{-} s}\right) \\
& \left.-R_{e d d y_{-} f} \cdot i_{e d d y_{-} f}\right) \\
\frac{\partial i_{e d d y_{-} s}=}{\partial t} & \frac{1}{L_{e d d y_{-} s}} \cdot\left(R_{e d d y_{-} f} \cdot i_{e d d y_{-} f}\right. \\
& \left.-R_{e d d y_{-} s} \cdot i_{e d d y_{-} s}\right)
\end{aligned}
$$

Main advantage of this model structure is its range of validity and the straightforward and automated parameter identification procedure easily realized on the basis of static FEM calculations for obtaining the well-known flux linkage $\Psi\left(i_{\text {coil }}, x_{\text {arm }}\right)$ and a very small number of dynamic computations for determination of 
eddy current parameters $R_{e d d y_{-} f, s}, L_{e d d y_{-} f, s}$.

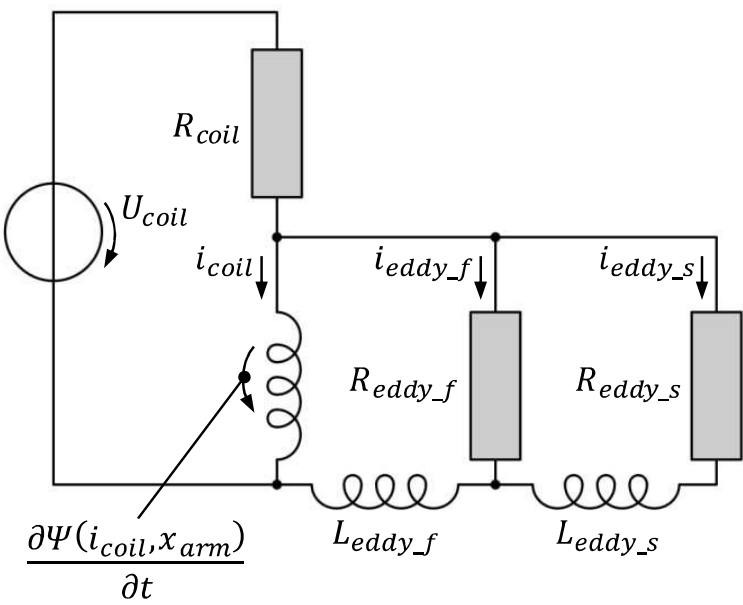

Figure 3: Equivalent electric circuit for proportional solenoid

Using the solenoid illustrated in Figure 2 and above mentioned implementation procedure the content of Figure 4 shows the obtained results in comparison with FEM calculations. The nonlinear differential inductivity is captured very well, also the saw-tooth-like distortion of the current signal in presence of eddy currents.

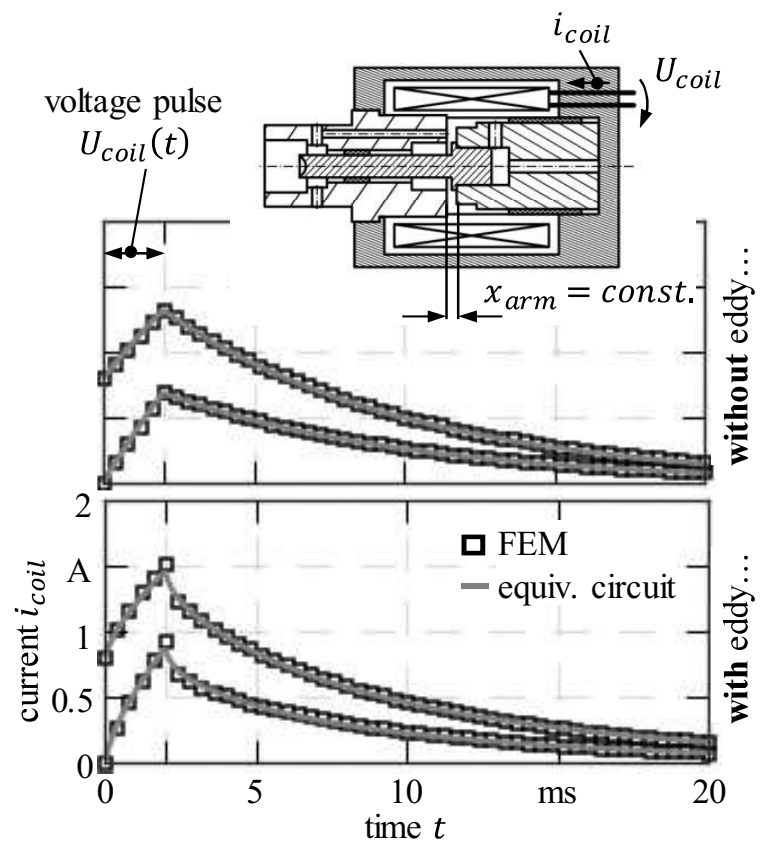

Figure 4: Verification of elaborated model structure

This model extended by a current controller for dither waveforms is well suited for the intended improvement of the reference valve design.

\subsection{Spool leakage}

In addition to the solenoid model, the leakage characteristic at the main spool plays an important role in obtaining a generally applicable hydraulic model of the entire system. The reasons are as various as the different test conditions and procedures for this type of valves. While the valve manufacturer typically tests with working port closed, the performance in clutch applications for example can be totally different. Main reason for this is the regularly used positive overlap at the main spool. This leads to miscellaneous operating conditions whereas the traveling range of the main spool either lies completely in the overlap region or partial opening at pressure and tank port happens.

The requirements for such a combined orificeleakage model arise on one hand from a mathematical point of view ensuring sufficiently smoothness and finiteness. On the other hand, the model approach has to guarantee a physically meaningful behavior. Finally, from the user's point of view, a geometrically based parameter selection should be possible.

The idea behind the utilized model is visualized in Figure 5, which is divided into three different stroke ranges for the spool depending on the geometrical overlap. In every spool stroke region parallel flow paths for orifice and leakage flow are present. Fluid flow based on orifice equation through the lateral surface at the bores opened by spool travelling is only existent in the "transition" and "open" state, otherwise it is blocked. 
complex flow \& leakage conditions
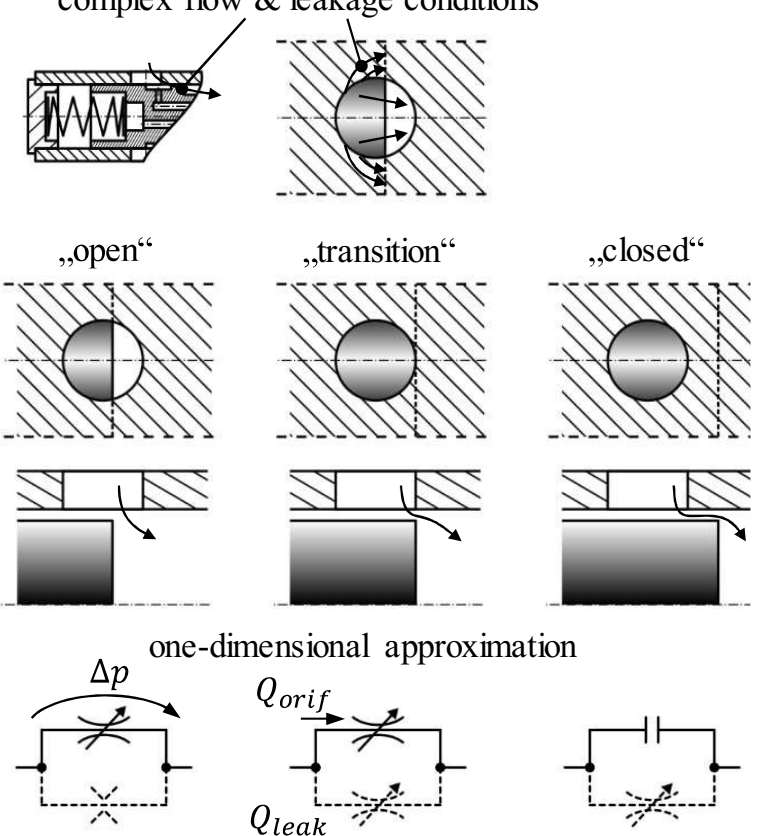

one-dimensional approximation
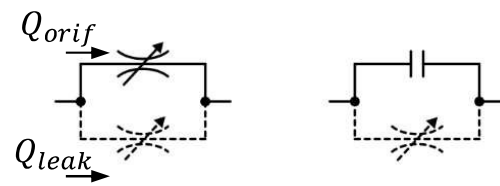

Figure 5: Hydraulic resistance network for fluid flow in different spool travelling regions

Besides the above mentioned regular modeling approach for hydraulic resistances, the parallel leakage path completes the model. This flow path guarantees that in operation situations with closed working port physical meaningful results are obtainable. Therefore, a change in flow rate with varying spool position is absolutely required. The modeling in the "closed" state follows the standard leakage equation whereas geometrical and modeled leakage lengths are shifted relative to each other. Because for zero leakage length fluid flow raises to infinity, some kind of limitation is inevitable. This is done by assuming orifice flow through the cross-sectional area of the leakage gap along the perimeter of the spool as sketched in Figure 5. This raw simplification depends only on geometrical parameters and is easily adjusted by scaling the area of the remaining leakage gap. A smooth crossover in the "transition" region between the "open" and "closed" state is ensured by interpolating the coefficient of resistance in a special manner as shown in Figure 6.

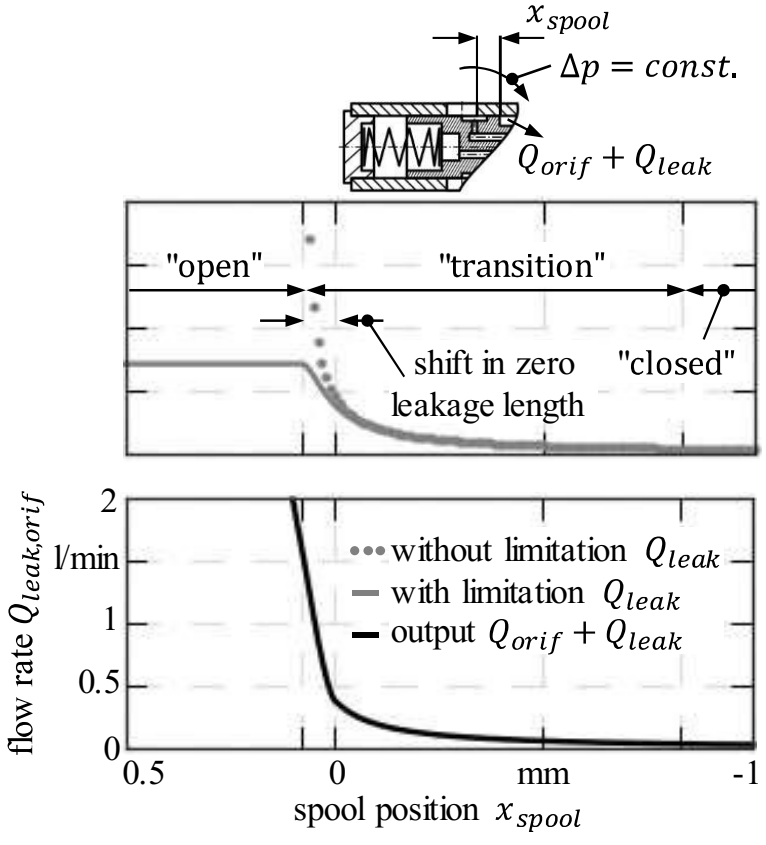

Figure 6: Exemplary simulation results for complete flow rate approximation

Like in the case of solenoid behavior, a better approximation can be achieved by incorporating additional results from numerical field simulation. Even without further effort, this model based on geometric data is more suitable than others for typical computations.

\subsection{Pressure-actuated clutch}

The final modeling challenge is to consider the customer's use case. This goes beyond standardized test procedures, but enables an application-oriented evaluation and adaption. A common simplification for a hydraulic clutch actuator is a spring-loaded piston. Here, the filling phase is emulated through the parameters piston area \& piston stroke. The load-transmitting behavior of the clutch plates leads to an additional micro-movement, which can be easily implemented via a non-linear spring or a nonlinear elastic end stop. Figure 7 illustrates the used model structure with a sketch of two underlying spring characteristics. 


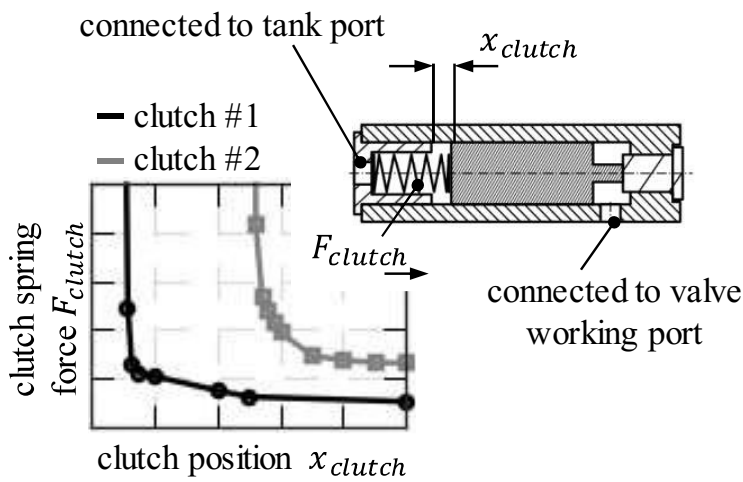

Figure 7: Simplification of pressure-actuated clutch

The parameters of the reduced model place pressure and flow requirements on the connected pressure control valve and have a direct effect on valve design and combined valve-clutch performance.

\section{REFERENCE DESIGN}

The valve behavior of a reference design is to serve as a starting point for further investigations. Beginning with the explanation of the inherent characteristics of the system, the performance evaluation differentiates between the valve alone and the additionally connected clutch. Measurements on a test bench are compared with the simulation results to confirm the application of the developed model for further simulationbased enhancements of the valve design.

\subsection{Quasi-static characterization}

Besides the flow capacity of the entire valve, the pressure signal characteristic curve is an important valve feature for reasons of interchangeability. The plotted curves in Figure 8 show a typical behavior observed in pressure control valves, whereas some main stage and clutch parameters can be read off the measurements. Under both boundary conditions, closed working port or with connected clutch, no functional anomalies occur.

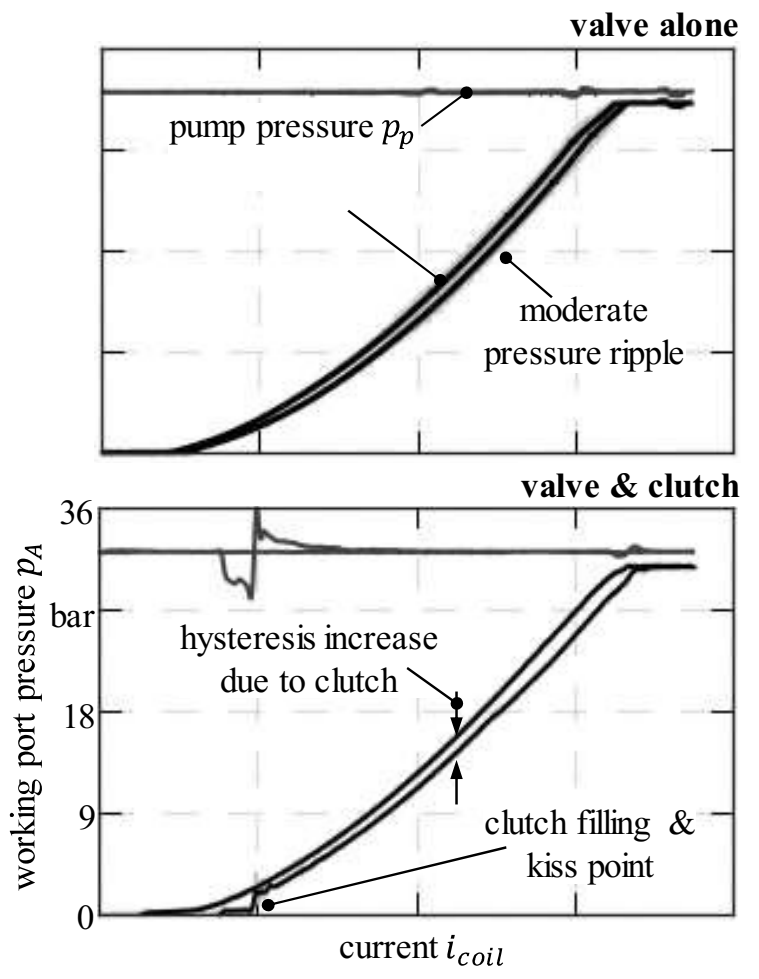

Figure 8: Measured characteristics of valve alone and valve with connected clutch under dither excitation

The static measurements could allow the conclusion that the valve used is suitable for clutch applications and test bench results of the valve alone are sufficient. However, a final evaluation requires at least the inclusion of an additional dynamic characterization.

\subsection{Transient input variations}

The dynamic investigations are based on typical control profiles for clutch valves as listed in [5]. Here step profiles are used for filling of the clutch and ramp profiles for load transfer. For ease of use, these profiles are separated into different step and ramp profiles and tested sequentially at the test bench. The Figures 9 - 10 show the obtained results clearly visualizing dynamic anomalies in clutch operation whereas valve alone behavior is completely unremarkable. 

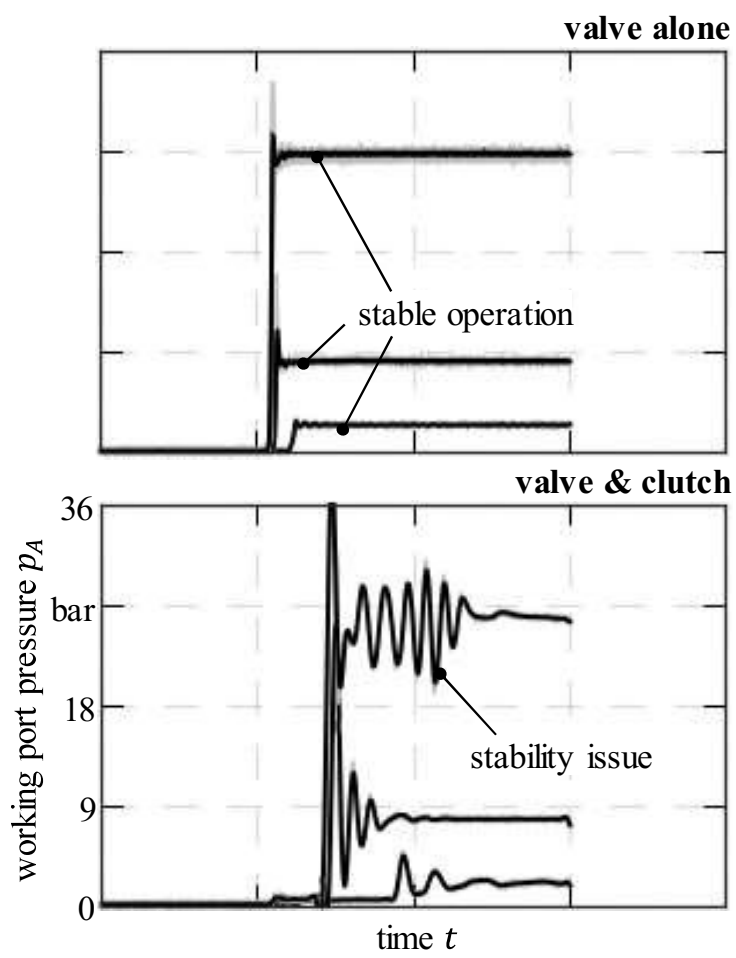

Figure 9: Measured step responses of valve alone and valve with connected clutch under dither excitation
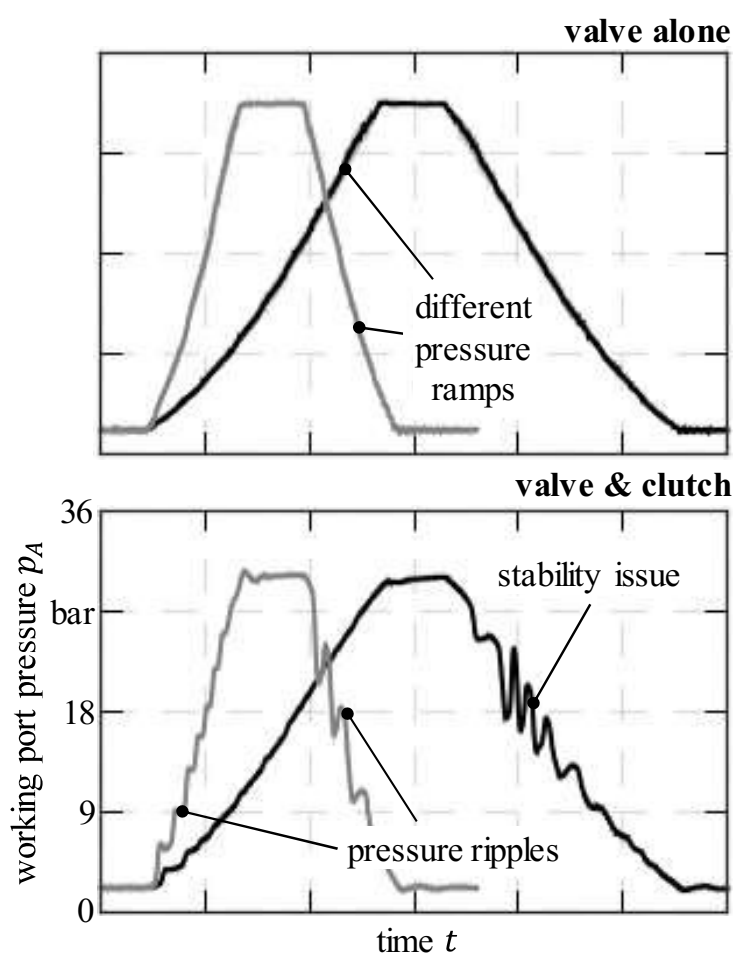

Figure 10: Measured ramp responses of valve alone and valve with connected clutch under dither excitation

Based on these results, valve selection neglecting the field of application can be misleading as well as designing valves without a detailed consideration of the application case during the development. This is a challenge for the valve manufacturers because the success partially depends on the cooperation with the system or machine supplier.

At this point the metrological characterization ends. The results show a significant difference between the different measurement conditions and motivate the subsequent simulation studies for the reference and next generation design.

\subsection{Simulation results}

Further development of pressure control valves based on simulation models is state-of-the-art in development departments, which is why the focus of this section is limited to some results of special interest.

Using the results from ramp profiles the necessity of the modified leakage model for an adequate calculation of valve performance is demonstrated. In the valve only case, during the ramp profile the main spool primarily operates in its dead band as illustrated in Figure 11.

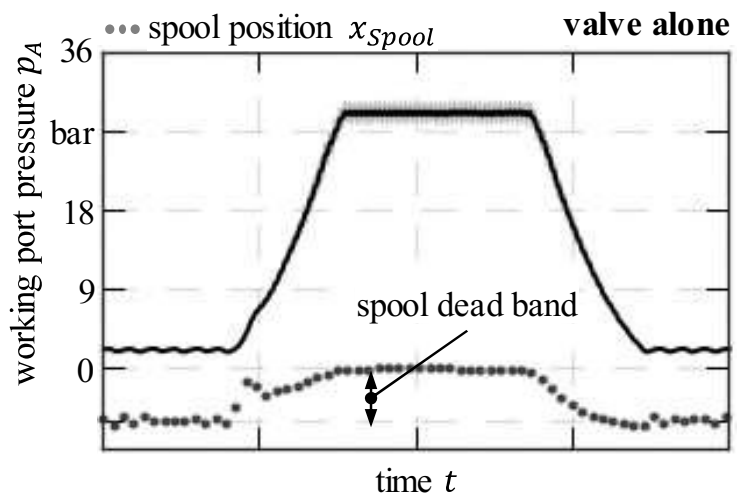

Figure 11: Simulation result for spool position during operation with closed working port

At the same time, Figure 11 also shows that the pressure ripple behavior and overall stability is in equivalence to the measurements for the valve only case.

Although the pressure fluctuations due to dither excitation are plausible, there is an essential point not governed by the simulation model. The lack of a dynamic hysteresis model leads to a disappearing hysteresis in the entire system regardless of the cause of hysteresis. However, since all measurements show a remaining hysteresis, an influence of this inadequacy on simulation accuracy can be 
suspected. This becomes particularly clear in the ramp behavior of the valve with connected clutch under dither and dither free current excitation as displayed in Figure 12. With dither excitation, the pressure build-up and release behavior of the pilot valve differs from dither free excitation resulting in a noticeably different transient response.

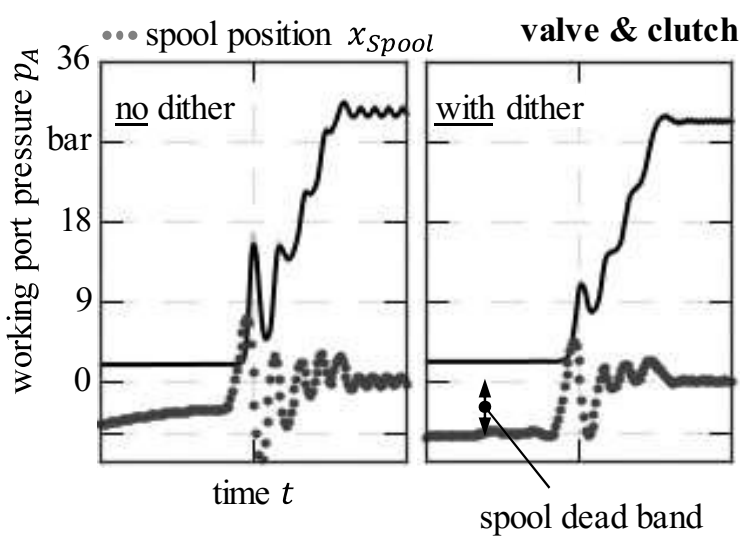

Figure 12: Influence of current control on simulated ramp behavior with connected clutch

As long as the model does not reflect this, deviations in simulated dynamics can be assumed. Another source of uncertainty is the simplified clutch with its not fully known properties.

The two previous examples illustrate the necessity for the modified leakage model to describe the flow rate behavior of the main spool and the solenoid model to map the dynamic force excitation at the pilot valve.

Further validation focusses on the ramp behavior because of its importance for clutch control. Figure 13 illustrates the final comparison between valve alone and valve with connected clutch. Differences in the simulation results are not as clearly visible as in the measurements in Figure 10. Nevertheless, problem areas can be identified in some regions of the transient response. Compared to measurements, the pressure fluctuations are smoothed out, whereby the cause can be the hysteresis behavior mentioned earlier or different or unknown clutch parameters.

With the present results, a starting point for further improvements of the reference design is given. The focus of the optimization is still the response behavior to transient input signal variations.
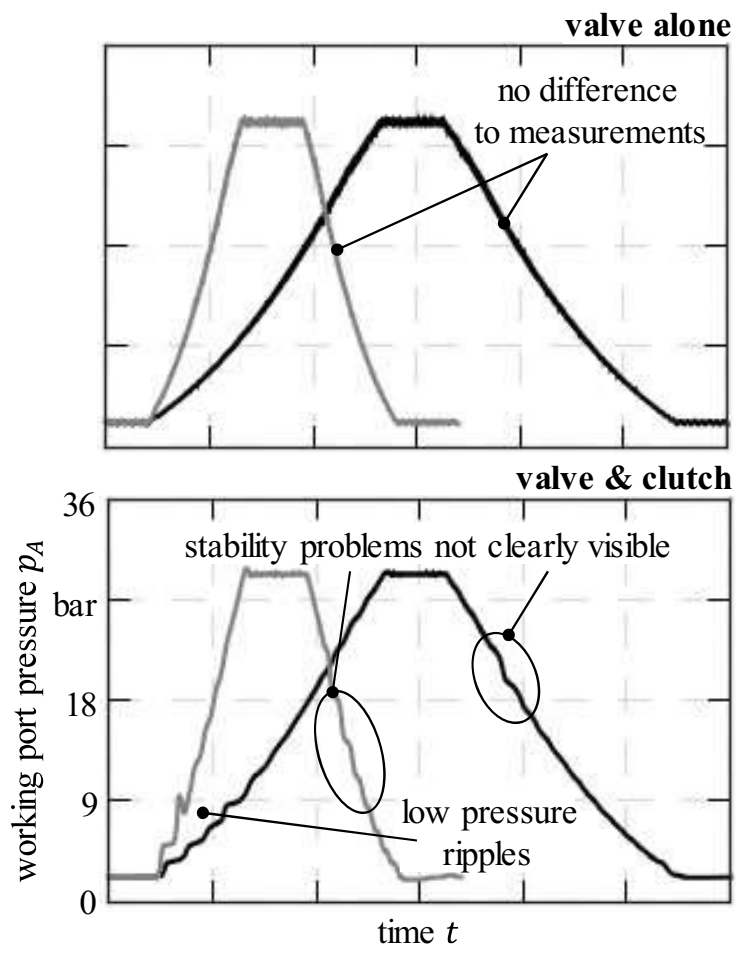

Figure 13: Simulated ramp behavior under dither excitation

\section{NEXT GENERATION DESIGN}

The aim of this section is to explain and present ideas for improving the dynamic response behavior. As mentioned in the previous section, the spool dead band, the dither excitation of the solenoid and the main spool travel influence the pressure characteristic and the pressure fluctuations. At the end, the suggestions for improvement are validated at the test bench.

Beginning with the pilot part of the clutch control valve from Figure 2, the pressure buildup and release during dither excitation is mainly determined by the amplification factor between opened flow surface and sphere travel. This results from the possible stroke changes due to dynamic solenoid force amplitudes during dither excitation. Another influence factor lies in the armature damping, here a middle way between stability and response time is essential.

At the main spool the situation is much more complex. On the one hand, the dead band of the main spool controls the leakage behavior but slows down the transient response by the time required for the opposite closing or opening of pump and tank port. On the other hand, spool response due to pressurization is influenced by spring forces and hydraulic counterforces from 
the opening and closing of the pressure and tank port. Care must be taken during adjustment of the main spool spring, as this will compromise interchangeability due to changes in the pressure signal characteristic curve.

The solution is achieved by targeted scanning of the solution space with plausible combinations of the underlying design variables or parameters. A major problem in the evaluation of every single solution were minor changes observed in the simulation result due to the filtering effect of the dither excitation.

Because the solution with closed working port is totally uncritical, afterwards only the simulation results with connected clutch are shown in Figure 14. Despite the only slight change in performance, an improvement is evident. For better visualization, the transient responses are shifted against each other by a small amount. The simulation results motivate the investigation of a hardware prototype to validate the computations and to confirm the interchangeability by means of pressure signal characteristic curve. Therefore, the measurements shown in Figures 8 - 10 are repeated under comparable operating conditions.

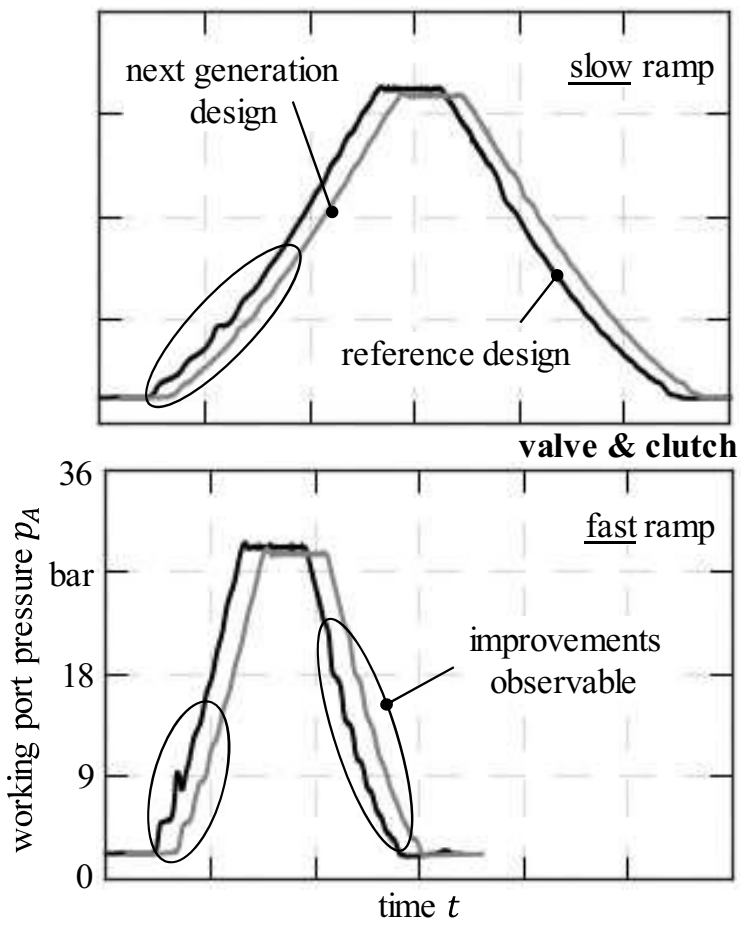

Figure 14: Ramp behavior with connected clutch under dither excitation after simulation-based optimization

The final measurement of the hardware prototype yields very promising results in contrast to the reference design. The ramp behavior shown in Figure 15 is completely uncritical and the comparability between valve alone and valve with connected clutch is almost given.

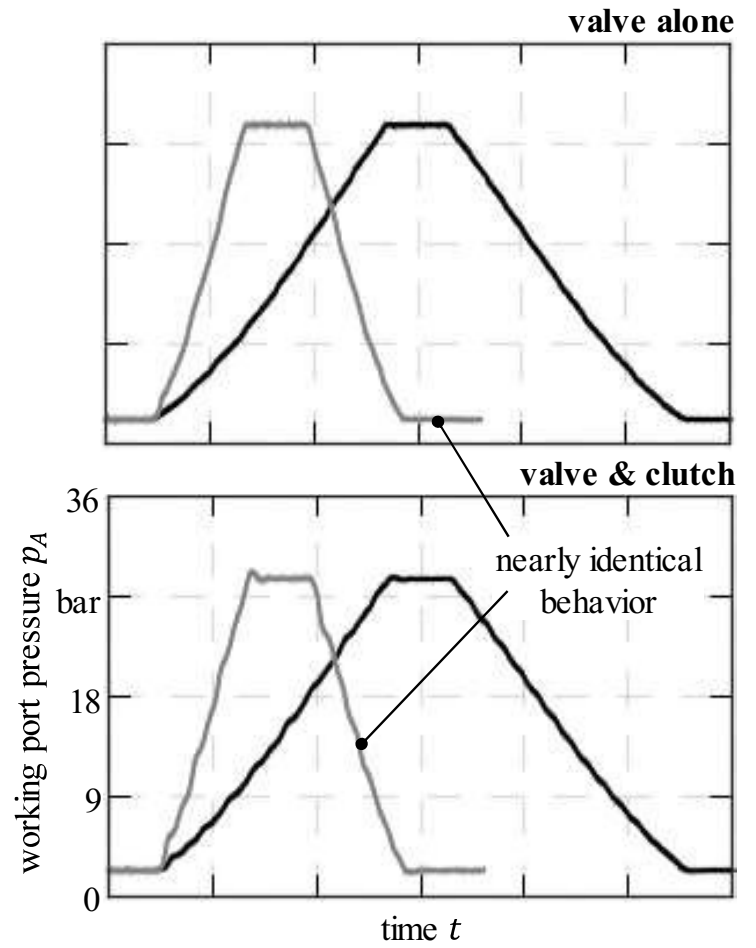

Figure 15: Measured ramp responses of valve alone and valve with connected clutch under dither excitation

A shortened summary of the remaining static and dynamic characterization is shown in Figure 16. In the upper part, the interchangeability between the reference and the next generation design is clearly visible. Additionally, an improvement in hysteresis is observable. The lower part visualizes the transient step responses. In comparison to Figure 9 a significant better damping is present and no anomalous vibrations occur.

The received results promise significantly better performance in the customer system by full interchangeability. At the same time the design could be simplified, which has a positive effect on assembly and costs.

\section{CONCLUSION}

The paper discussed two important modeling challenges for investigated pilot-operated pressure reducing valve used in clutch control applications. It was shown, that spool leakage dominates the static and transient system 
behavior in the case of closed working port. The necessity of an accurate solenoid model resulted from the dither excitation used. Without consideration of the clutch, valve optimization was not possible because of missing operating failures as a starting point for improvements.

Despite the slightly difficult comparison of the simulation results an improved solution could be found. Measurements confirmed the predictions and encouraged the approach chosen. Some modeling issues remained unanswered. This applies to the dynamic hysteresis and the clutch modeling and its parameters. These uncertainties provide a basis for further investigations.

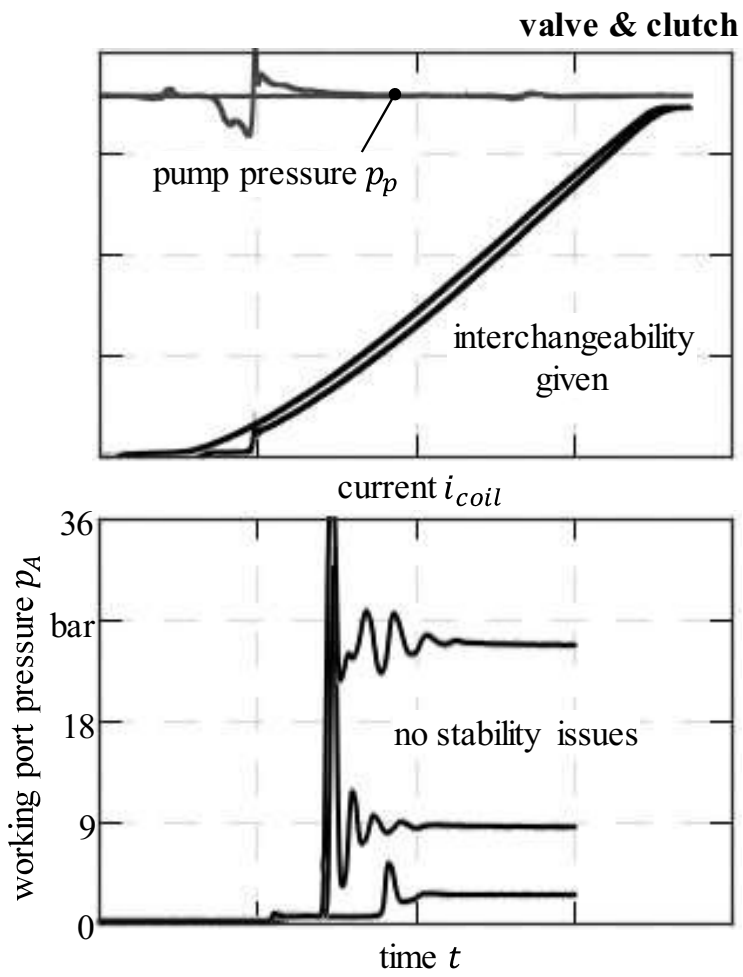

Figure 16: Measured characteristic curve and step responses of valve with connected clutch under dither excitation

\section{NOMENCLATURE}

$F_{\text {arm }}$ Armature force [N]

$F_{\text {clutch }}$ Clutch counter force [N]

$i_{\text {eddy }}$ Eddy current [A]

$i_{\text {coil }}$ Coil current $[\mathrm{A}]$

$L_{e d d y}$ Eddy inductance [H]

$\Delta p \quad$ Pressure difference [bar]

$p_{A} \quad$ Working port pressure [bar]

$p_{P} \quad$ Pump pressure [bar]

$p_{T} \quad$ Tank pressure [bar]

$Q_{\text {leak }} \quad$ Leakage flow rate $[1 / \mathrm{min}]$
$Q_{\text {orif }}$ Orifice flow rate $[1 / \mathrm{min}]$

$R_{\text {coil }} \quad$ Coil resistance $[\Omega]$

$R_{\text {eddy }}$ Eddy resistance [ $\Omega$ ]

$t \quad$ Time [s]

$U_{\text {coil }}$ Coil voltage [V]

$\vec{x} \quad$ State variable [-]

$x_{\text {arm }}$ Armature position [mm]

$x_{\text {clutch }}$ Clutch position [mm]

$x_{\text {spool }}$ Spool position [mm]

$\Psi \quad$ Flux linkage [Wb]

\section{REFERENCES}

[1] Cristofori A, Vacca A (2012) The Modeling of Electrohydraulic Proportional Valves. J Dyn Sys Meas Control. 134(2):021008

[2] Kallenbach E, Ströhla T (2002) Dynamic Simulation of Electromagnetic Actuators using Network Models Including Eddy Currents. In: Proc $8^{\text {th }}$ Int Conf New Actuators (ACTUATOR 2002), Bremen, 12-14 June 2002

[3] Vaughan N D, Gamble J B (1996) The Modeling and Simulation of Proportional Solenoid Valve. J Dyn Sys Meas Control. 118(1):120-125

[4] Biller H (2008) Dynamic Simulation of Electromagnets. In: Proc. European Comsol Conference, Hannover, 4-6 November 2008

[5] Schmidt T et.al. (2005) Verfahren zum Steuern und Regeln eines Automatikgetriebes eines Fahrzeugs. DE102005042933A1, 9 Sept 2005 